\title{
On Hardy's Inequality for Hermite Expansions
}

\author{
Paweł Plewa
}

\begin{abstract}
Sharp multi-dimensional Hardy's inequality for the Laguerre functions of Hermite type is proved for the type parameter $\alpha \in[-1 / 2, \infty)^{d}$. As a consequence we obtain the corresponding result for the generalized Hermite expansions. In particular, it validates that the known version of Hardy's inequality for the Hermite functions is sharp.
\end{abstract}

\section{Introduction}

Hardy and Littlewood [4] proved the following inequality for Fourier coefficients

$$
\sum_{k \in \mathbb{Z}} \frac{|\widehat{f}(k)|}{|k|+1} \lesssim\|f\|_{\operatorname{Re} H^{1}}
$$

where Re $H^{1}$ denotes the real Hardy space constituted by the boundary values of the real parts of functions in the Hardy space $H^{1}(\mathbb{D})$ with $\mathbb{D}$ the unit disk in the plane.

Kanjin [5] initiated investigation of analogues of (1.1) for orthogonal expansions. He proved the one-dimensional version of the following inequality

$$
\sum_{n \in \mathbb{N}^{d}} \frac{\left|\left\langle f, h_{n}\right\rangle\right|}{\left(n_{1}+\cdots+n_{d}+1\right)^{E}} \lesssim\|f\|_{H^{1}\left(\mathbb{R}^{d}\right)}, \quad f \in H^{1}\left(\mathbb{R}^{d}\right),
$$

where $n=\left(n_{1}, \ldots, n_{d}\right),\langle\cdot, \cdot\rangle$ stands for the inner product in $L^{2}\left(\mathbb{R}^{d}\right),\left\{h_{n}\right\}_{n \in \mathbb{N}^{d}}$ are the Hermite functions, and $H^{1}\left(\mathbb{R}^{d}\right)$ denotes the Hardy space. We will refer to the constant $E$ as the admissible exponent.

Recently many authors studied Hardy's inequality for Hermite expansions. In the mentioned article Kanjin examined only the case $d=1$ and proved a version of 1.2 , with $E=29 / 36$. Later Radha 14 investigated the multi-dimensional setting $d \geq 1$. For an arbitrary $\varepsilon>0$, the admissible exponent $E=(17 d+12+\varepsilon) /(12 d+24)$ was obtained. Then Radha and Thangavelu [15] received $E=3 d / 4$ for $d \geq 2$. Unfortunately, the applied method did not work in the one-dimensional case. Kanjin [6] basing on a

Received January 17, 2019; Accepted June 2, 2019.

Communicated by Duy-Minh Nhieu.

2010 Mathematics Subject Classification. Primary: 42C10; Secondary: 42B30, 33C45.

Key words and phrases. Hardy's inequality, Hardy's space, Hermite expansions, Laguerre expansions of Hermite type. 
paper of Balasubramanian and Radha 2 justified that for $d=1$ the admissible exponent is $E=3 / 4+\varepsilon$, for an arbitrary $\varepsilon>0$. He also conjectured that it can be lowered to $3 / 4$. It was indeed proved by Z. Li, Y. Yu and Y. Shi 9 .

Hardy's inequality was also investigated in the context of different orthonormal expansions as well. Kanjin and Sato [7] studied the case of the Jacobi expansions. Moreover, the author considered various Laguerre expansions in 12,13. Furthermore, an analogue of Hardy's inequality was scrutinized, namely the Hardy space $H^{1}$ was replaced by $H^{p}$ for $p \in(0,1)$ (see $2,15,16])$.

The primary goal of this article is to prove that the admissible exponent in $(1.2)$ cannot be lowered. For this purpose we extend the result from [12] for Laguerre expansions of Hermite type, to a wider range of the type parameter, namely $\alpha \in[-1 / 2, \infty)^{d}$. We also construct an explicit counterexample to show that the associated admissible exponent $E=3 d / 4$ is sharp. Moreover, we are able to deduce the corresponding result for the generalized Hermite expansions along with its sharpness. Consequently, we get sharpness of 1.2 with $E=3 d / 4$.

Our main tool in establishing Hardy's inequality is [13, Theorem 2.2]. The verification of the required conditions for the type parameter $\alpha \in(-1 / 2,1 / 2)$ is more complicated than for $\alpha \in\{-1 / 2\} \cup[1 / 2, \infty)$ (as it was implicitly done in [12]). In order to deduce Hardy's inequality for the generalized Hermite setting from the result for the Laguerre setting of Hermite type, we apply a decomposition of functions on $\mathbb{R}^{d}$ with respect to its parity. Using the same method one can prove an $L^{1}$-analogue of Hardy's inequality (compare 6, 12,13).

The organization of this paper is as follows. In Section 2 we state preliminaries, mainly some facts about the Hardy spaces, and recall [13, Theorem 2.2]. Section 3 is devoted to the Laguerre expansions of Hermite type. We present some auxiliary results leading to the verification of the assumptions of Theorem 2.2. Furthermore, we construct the mentioned counterexample. In Section 4 we justify that Hardy's inequality for the generalized Hermite expansions follows from the corresponding result for the Laguerre functions of Hermite type.

Notations. Throughout this paper we shall denote $\mathbb{R}_{+}^{d}=(0, \infty)^{d}$ and $\mathbb{N}_{+}=\mathbb{N} \backslash\{0\}=$ $\{1,2, \ldots\}$, where $d \geq 1$ is the dimension. We shall distinguish the one-dimensional variables from the multi-dimensional ones. Therefore, in the case $d=1$ we write $u, v$ for real variables and $k$ or $j$ for non-negative integers. On the other hand, in the case $d \geq 1$ we use $x=\left(x_{1}, \ldots, x_{d}\right), y=\left(y_{1}, \ldots, y_{d}\right)$ for real vectors, and $n=\left(n_{1}, \ldots, n_{d}\right) \in \mathbb{N}^{d}$ for multi-indices. The Euclidean norm is denoted by $|x|$ and $|y|$, whereas $|n|=n_{1}+\cdots+n_{d}$ stands for the length of $n$. If a multi-index is constant, then we will use the bold font, e.g., $\mathbf{0}=(0, \ldots, 0)$. The Laguerre type multi-index $\alpha=\left(\alpha_{1}, \ldots, \alpha_{d}\right) \in(-1, \infty)^{d}$ will be 
denoted by the same symbol in both cases $d=1$ and $d \geq 1$. It should be always clear from the context whether $\alpha$ refers to $d=1$ or $d \geq 1$. Similarly as before, $|\alpha|=\alpha_{1}+\cdots+\alpha_{d}$ stands for the length of the multi-index $\alpha$. Note that $|\alpha|$ may be negative. We will use the usual convention writing $x^{\alpha}=\prod_{i=1}^{d} x_{i}^{\alpha_{i}}, x \in \mathbb{R}_{+}^{d}$. If a function $f$ is defined on $\mathbb{R}^{d}$, then its restriction to $\mathbb{R}_{+}^{d}$ is denoted by $f^{+}$.

The symbol $\lesssim$ stands for inequalities that hold with a multiplicative constant that may vary from line to line. Such a constant may depend on parameters quantified beforehand, but not on the ones quantified afterwards. If $\lesssim$ and $\gtrsim$ hold simultaneously, then we will write $\simeq$.

\section{Preliminaries}

A measurable function $f$ defined on $\mathbb{R}^{d}$ is called $\eta$-symmetric for some $\eta \in\{0,1\}^{d}$, if $f$ is even with respect to every $i$-th coordinate such that $\eta_{i}=0$ and odd with respect to the remaining coordinates. We shall make use of the decomposition

$$
f=\sum_{\eta \in\{0,1\}^{d}} f_{\eta}
$$

where

$$
f_{\eta}(x)=2^{-d} \sum_{\epsilon \in\{-1,1\}^{d}} \epsilon^{\eta} f(\epsilon x) .
$$

The classical Hardy space $H^{1}\left(\mathbb{R}^{d}\right)$ can be defined in many ways (see [17]), e.g., given a Schwartz function $\psi$ such that $\int \psi \neq 0$, we say that a function $f \in L^{1}\left(\mathbb{R}^{d}\right)$ belongs to $H^{1}\left(\mathbb{R}^{d}\right)$ if and only if

$$
\|f\|_{H_{m}^{1}\left(\mathbb{R}^{d}\right)}:=\left\|\sup _{t>0}\left|f * \psi_{t}\right|\right\|_{L^{1}\left(\mathbb{R}^{d}\right)}<\infty,
$$

where $\psi_{t}(x)=t^{-d} \psi(x / t)$. The definition of $H^{1}\left(\mathbb{R}^{d}\right)$ is independent of the chosen function $\psi$. The definition (2.1) is referred to as the maximal characterization of $H^{1}\left(\mathbb{R}^{d}\right)$. We emphasize that

$$
\|f\|_{L^{1}\left(\mathbb{R}^{d}\right)} \lesssim\|f\|_{H_{m}^{1}\left(\mathbb{R}^{d}\right)}
$$

A measurable function $a$ is called an $H^{1}\left(\mathbb{R}^{d}\right)$-atom if it is supported in a Euclidean ball $B$ and satisfies the cancellation condition and the size condition, namely $\int a(x) d x=0$ and $\|a\|_{L^{\infty}\left(\mathbb{R}^{d}\right)} \leq|B|^{-1}$, respectively, where $|B|$ denotes the Lebesgue measure of $B$.

A function $f$ is in $H^{1}\left(\mathbb{R}^{d}\right)$ if and only if it admits an atomic decomposition, i.e., there exist a sequence of coefficients $\left\{\lambda_{j}\right\}_{j \in \mathbb{N}}$ and a sequence of $H^{1}\left(\mathbb{R}^{d}\right)$-atoms $\left\{a_{j}\right\}_{j \in \mathbb{N}}$ such that

$$
f(x)=\sum_{j=0}^{\infty} \lambda_{j} a_{j}(x)
$$


where the series is convergent in $H^{1}\left(\mathbb{R}^{d}\right)$. Moreover,

$$
\sum_{j=0}^{\infty}\left|\lambda_{j}\right| \lesssim\|f\|_{H_{m}^{1}\left(\mathbb{R}^{d}\right)}
$$

We define

$$
\|f\|_{H_{a t}^{1}\left(\mathbb{R}^{d}\right)}=\inf \sum_{j=0}^{\infty}\left|\lambda_{j}\right|
$$

where the the infimum is taken over all atomic decompositions of $f$. The norms $\|\cdot\|_{H_{m}^{1}\left(\mathbb{R}^{d}\right)}$ and $\|\cdot\|_{H_{a t}^{1}\left(\mathbb{R}^{d}\right)}$ are equivalent. From now on, we shall use the latter and write simply $\|\cdot\|_{H^{1}\left(\mathbb{R}^{d}\right)}$.

We emphasise that for $f \in H^{1}\left(\mathbb{R}^{d}\right)$ and every $\epsilon \in\{-1,1\}^{d}$ we have $\|f\|_{H^{1}\left(\mathbb{R}^{d}\right)}=$ $\|f(\epsilon \cdot)\|_{H^{1}\left(\mathbb{R}^{d}\right)}$. Hence, for any $\eta \in\{0,1\}^{d}$ there is $f_{\eta} \in H^{1}\left(\mathbb{R}^{d}\right)$ and $\left\|f_{\eta}\right\|_{H^{1}\left(\mathbb{R}^{d}\right)} \leq$ $\|f\|_{H^{1}\left(\mathbb{R}^{d}\right)}$.

The following lemma holds.

Lemma 2.1. If $\eta \in\{0,1\}^{d}$ and $f \in H^{1}\left(\mathbb{R}^{d}\right)$ is $\eta$-symmetric, then $f \mathbb{1}_{\mathbb{R}_{+}^{d}} \in H^{1}\left(\mathbb{R}^{d}\right)$. Moreover,

$$
\left\|f \mathbb{1}_{\mathbb{R}_{+}^{d}}\right\|_{H^{1}\left(\mathbb{R}^{d}\right)} \simeq\|f\|_{H^{1}\left(\mathbb{R}^{d}\right)} .
$$

Proof. Fix $\eta \in\{0,1\}^{d}$ and $\eta$-symmetric function $f \in H^{1}\left(\mathbb{R}^{d}\right)$. We choose an atomic decomposition of $f$. Let

$$
f(x)=\sum_{j=0}^{\infty} \lambda_{j} a_{j}(x)
$$

where $a_{j}$ 's are $H^{1}\left(\mathbb{R}^{d}\right)$-atoms. Hence,

$$
f(x) \mathbb{1}_{\mathbb{R}_{+}^{d}}(x)=f_{\eta}(x) \mathbb{1}_{\mathbb{R}_{+}^{d}}(x)=\sum_{j=0}^{\infty} \lambda_{j} 2^{-d} \sum_{\epsilon \in\{-1,1\}^{d}} \epsilon^{\eta} a_{j}(\epsilon x) \mathbb{1}_{\mathbb{R}_{+}^{d}}(x) .
$$

In order to prove that $\left\|f \mathbb{1}_{\mathbb{R}_{+}^{d}}\right\|_{H^{1}\left(\mathbb{R}^{d}\right)} \leq\|f\|_{H^{1}\left(\mathbb{R}^{d}\right)}$ it suffices to justify that for any $H^{1}\left(\mathbb{R}^{d}\right)$-atom $a$, the function

$$
a_{\eta}(x) \mathbb{1}_{\mathbb{R}_{+}^{d}}(x)=2^{-d} \sum_{\epsilon \in\{-1,1\}^{d}} \epsilon^{\eta} a(\epsilon x) \mathbb{1}_{\mathbb{R}_{+}^{d}}(x)
$$

is an $H^{1}\left(\mathbb{R}^{d}\right)$-atom as well. Indeed, if the inferior of the support of $a$ does not intersect any of the hyperplanes $\left\langle e_{i}\right\rangle^{\perp}, i=1, \ldots, d$, then for all but one $\epsilon \in\{-1,1\}^{d}$, there is $a(\epsilon \cdot) \mathbb{1}_{\mathbb{R}_{+}^{d}} \equiv 0$. For the remaining $\epsilon$ there holds $a(\epsilon \cdot) \mathbb{1}_{\mathbb{R}_{+}^{d}}=\epsilon^{\eta} a$, so $a_{\eta} \mathbb{1}_{\mathbb{R}_{+}^{d}}$ is an $H^{1}\left(\mathbb{R}^{d}\right)$ atom.

Let us now define

$$
I=\left\{i \in\{1, \ldots, d\}: \operatorname{int} \operatorname{supp} a \cap\left\langle e_{i}\right\rangle^{\perp} \neq \emptyset\right\}
$$


where int denotes the interior of a set. Without loss of generality we may assume that we have $I=\{1, \ldots, k\}$ for some $k \in\{1, \ldots, d\}$. Then, for any $\epsilon_{2} \in\{-1,1\}^{d-k}$ the function

$$
2^{-k}\left(\sum_{\epsilon_{1} \in\{-1,1\}^{k}}\left(\epsilon_{1}, \epsilon_{2}\right)^{\eta} a\left(\left(\epsilon_{1}, \epsilon_{2}\right) x\right)\right) \mathbb{1}_{\mathbb{R}_{+}^{d}}(x)
$$

is an $H^{1}\left(\mathbb{R}^{d}\right)$-atom. Moreover, for all but one $\epsilon_{2} \in\{-1,1\}^{d-k}$ the function above vanishes identically. Therefore $a_{\eta}(x) \mathbb{1}_{\mathbb{R}_{+}^{d}}(x)$ is an $H^{1}\left(\mathbb{R}^{d}\right)$-atom. Hence, $f \mathbb{1}_{\mathbb{R}_{+}^{d}} \in H^{1}\left(\mathbb{R}^{d}\right)$ and

$$
\left\|f \mathbb{1}_{\mathbb{R}_{+}^{d}}\right\|_{H^{1}\left(\mathbb{R}^{d}\right)} \leq\|f\|_{H^{1}\left(\mathbb{R}^{d}\right)}
$$

In order to justify the opposite estimate we notice that

$$
\|f\|_{H^{1}\left(\mathbb{R}^{d}\right)}=\left\|\sum_{\epsilon \in\{-1,1\}^{d}} f(\epsilon \cdot) \mathbb{1}_{\mathbb{R}_{+}^{d}}\right\|_{H^{1}\left(\mathbb{R}^{d}\right)}=\left\|\sum_{\epsilon \in\{-1,1\}^{d}} \epsilon^{\eta} f \mathbb{1}_{\mathbb{R}_{+}^{d}}\right\|_{H^{1}\left(\mathbb{R}^{d}\right)} \leq 2^{d}\left\|f \mathbb{1}_{\mathbb{R}_{+}^{d}}\right\|_{H^{1}\left(\mathbb{R}^{d}\right)} .
$$

This finishes the proof of the lemma.

We define the Hardy space $H^{1}\left(\mathbb{R}_{+}^{d}\right)$ as follows. A function $f \in L^{1}\left(\mathbb{R}_{+}^{d}\right)$ belongs to $H^{1}\left(\mathbb{R}_{+}^{d}\right)$ if there exists $g \in H^{1}\left(\mathbb{R}^{d}\right)$ such that $\operatorname{supp} g \subset[0, \infty)^{d}$ and $g^{+}=f$. Moreover, we set $\|f\|_{H^{1}\left(\mathbb{R}_{+}^{d}\right)}=\|g\|_{H^{1}\left(\mathbb{R}^{d}\right)}$.

The proof of Lemma 2.1 yields that $f \in H^{1}\left(\mathbb{R}_{+}^{d}\right)$ if and only if it admits an atomic decomposition as in $(2.2)$, where $a_{j}$ are $H^{1}\left(\mathbb{R}_{+}^{d}\right)$-atoms, e.g., $a_{j}$ are usual atoms and their supports are Euclidean balls intersected with $[0, \infty)^{d}$. Furthermore, for $\eta \in\{0,1\}^{d}$ and $f \in H^{1}\left(\mathbb{R}^{d}\right)$ there is

$$
\left\|f_{\eta}^{+}\right\|_{H^{1}\left(\mathbb{R}_{+}^{d}\right)} \simeq\left\|f_{\eta}\right\|_{H^{1}\left(\mathbb{R}^{d}\right)} \leq\|f\|_{H^{1}\left(\mathbb{R}^{d}\right)} .
$$

We shall make use of [13, Theorem 2.2]. For the reader's convenience we state it below (only for Lebesgue measure).

Theorem 2.2. Let $X$ be an open convex subset of $\mathbb{R}^{d}$. For a given orthonormal basis $\left\{\varphi_{n}\right\}_{n \in \mathbb{N}^{d}}$ in $L^{2}(X)$, such that $\varphi_{n} \in L^{\infty}(X), n \in \mathbb{N}^{d}$, we define a family of operators $\left\{R_{r}\right\}_{r \in(0,1)}$ via

$$
R_{r} f=\sum_{n \in \mathbb{N}^{d}} r^{|n|}\left\langle f, \varphi_{n}\right\rangle \varphi_{n}, \quad r \in(0,1), f \in L^{2}(X) .
$$

We assume that the operators $R_{r}$ are integral operators and the associated kernels satisfy for some $\gamma>0$ and a finite set $\Delta$ composed of positive numbers the condition

$$
\left\|R_{r}(x, \cdot)-R_{r}\left(x^{\prime}, \cdot\right)\right\|_{L^{2}(X)} \lesssim \sum_{\delta \in \Delta}\left|x-x^{\prime}\right|^{\delta}(1-r)^{-\gamma(d+2 \delta) /(d+2)}
$$


uniformly in $r \in(0,1), x^{\prime} \in X$, and almost every $x$ such that $\left|x^{\prime}-x\right| \leq 1 / 3$. Then the inequality

$$
\sum_{n \in \mathbb{N}^{d}} \frac{\left|\left\langle f, \varphi_{n}\right\rangle\right|}{(|n|+1)^{E}} \lesssim\|f\|_{H^{1}(X)}
$$

holds uniformly in $f \in H^{1}(X)$, where

$$
E=\frac{\gamma d}{d+2}+\frac{d}{2}
$$

In the theorem above the space $H^{1}(X)$ is a Hardy space is the sense of Coifman-Weiss (see [3, pp. 591-592]). If $X=\mathbb{R}^{d}$ or $X=\mathbb{R}_{+}^{d}$, then it coincides with the definitions presented before.

\section{Laguerre functions of Hermite type}

The Laguerre functions of Hermite type are defined by the formula

$$
\varphi_{k}^{\alpha}(u)=\left(\frac{2 \Gamma(k+1)}{\Gamma(k+\alpha+1)}\right)^{1 / 2} L_{k}^{\alpha}\left(u^{2}\right) u^{\alpha+1 / 2} e^{-u^{2} / 2}, \quad u>0,
$$

in the one-dimensional case, and as the tensor product in higher dimensions. The system of functions $\left\{\varphi_{n}^{\alpha}\right\}_{n \in \mathbb{N}^{d}}$ is an orthonormal basis in $L^{2}\left(\mathbb{R}_{+}^{d}\right)$.

We will make use of the known estimates (see [10, p. 435] and [1, p. 699])

$$
\left|\varphi_{k}^{\alpha}(u)\right| \lesssim \begin{cases}u^{\alpha+1 / 2} \nu^{\alpha / 2} & \text { if } 0<u \leq 1 / \sqrt{\nu} \\ \nu^{-1 / 4} & \text { if } 1 / \sqrt{\nu}<u \leq \sqrt{\nu / 2} \\ u^{1 / 2}\left(\nu\left(\nu^{1 / 3}+\left|u^{2}-\nu\right|\right)\right)^{-1 / 4} & \text { if } \sqrt{\nu / 2}<u \leq \sqrt{3 \nu / 2} \\ u^{1 / 2} \exp \left(-\gamma u^{2}\right) & \text { if } \sqrt{3 \nu / 2}<u<\infty,\end{cases}
$$

where $\nu=\nu(\alpha, k)=\max (4 k+2 \alpha+2,2)$ and with $\gamma>0$ depending only on $\alpha$.

Using (3.1) for $\alpha \geq-1 / 2$ one gets

$$
\left\|\varphi_{k}^{\alpha}\right\|_{L^{\infty}(\mathbb{R})} \lesssim(k+1)^{-1 / 12}, \quad\left\|\varphi_{k}^{\alpha}\right\|_{L^{\infty}((0,1))} \lesssim(k+1)^{-1 / 4}, \quad k \in \mathbb{N},
$$

compare [18, p. 99]. Moreover, using (3.1) and the recurrence formula

$$
\frac{d}{d u} \varphi_{k}^{\alpha}(u)=-2 \sqrt{k} \varphi_{k-1}^{\alpha+1}(u)+\left(\frac{2 \alpha+1}{2 u}-u\right) \varphi_{k}^{\alpha}(u),
$$

where $\varphi_{-1}^{\alpha} \equiv 0$, we obtain for $\alpha \in\{-1 / 2\} \cup[1 / 2, \infty)$,

$$
\left\|\frac{d}{d \cdot} \varphi_{k}^{\alpha}(\cdot)\right\|_{L^{\infty}(\mathbb{R})} \lesssim(k+1)^{5 / 12}, \quad k \in \mathbb{N} .
$$


The estimate fails to hold for $\alpha \in(-1 / 2,1 / 2)$. However, it is easy to prove that for $\alpha \in[-1 / 2, \infty)$ we have

$$
\left\|\frac{d}{d \cdot} \varphi_{k}^{\alpha}(\cdot)\right\|_{L^{\infty}([1 / 2, \infty))} \lesssim(k+1)^{5 / 12}, \quad k \in \mathbb{N} .
$$

In order to prove Hardy's inequality associated with the Laguerre functions of Hermite type we shall use Theorem 2.2. The kernels associated with the family of integral operators $\left\{R_{r}^{\alpha}\right\}_{r \in(0,1)}$ for Laguerre functions of Hermite type, defined as in (2.4), are of the form

$$
R_{r}^{\alpha}(x, y)=\sum_{n \in \mathbb{N}^{d}} r^{|n|} \varphi_{n}^{\alpha}(x) \varphi_{n}^{\alpha}(y), \quad x, y \in \mathbb{R}_{+}^{d},
$$

and, for $d=1$, can be explicitly expressed by (compare [19, p. 102])

$$
R_{r}^{\alpha}(u, v)=\frac{2(u v)^{1 / 2}}{(1-r) r^{\alpha / 2}} \exp \left(-\frac{1+r}{2(1-r)}\left(u^{2}+v^{2}\right)\right) I_{\alpha}\left(\frac{2 r^{1 / 2}}{1-r} u v\right),
$$

where $I_{\alpha}$ denotes the modified Bessel function of the first kind, and as the tensor product in higher dimensions.

We remark that in the light of [12, Lemma 3.1] in order to verify the multi-dimensional assumption 2.5) (with $\gamma=-(d+2) / 4$ and $\Delta=\left\{1, \alpha_{1}+1 / 2, \ldots, \alpha_{d}+1 / 2\right\}$, excluding zeros) for the Laguerre functions of Hermite type with $\alpha \in[-1 / 2, \infty)^{d}$, it suffices to prove the following one-dimensional result.

Proposition 3.1. If $\alpha \in[-1 / 2, \infty)$, then

$$
\left\|R_{r}^{\alpha}(u, \cdot)-R_{r}^{\alpha}\left(u^{\prime}, \cdot\right)\right\|_{L^{2}\left(\mathbb{R}_{+}\right)} \lesssim \frac{\left|u-u^{\prime}\right|}{(1-r)^{3 / 4}}+\frac{\left|u-u^{\prime}\right|^{\alpha+1 / 2}}{(1-r)^{(\alpha+1) / 2}}
$$

uniformly in $r \in(0,1)$ and $u, u^{\prime}>0$ such that $\left|u-u^{\prime}\right| \leq 1 / 2$. If $\alpha=-1 / 2$, then we omit the second component on the right-hand side of the estimate.

Before the proof of the proposition we present two auxiliary lemmas.

Lemma 3.2. If $\alpha \in(-1 / 2,1 / 2)$, then

$$
\left|\varphi_{k}^{\alpha}(u)-\varphi_{k}^{\alpha}(v)\right| \lesssim|u-v|(k+1)^{-1 / 4}+|u-v|^{\alpha+1 / 2}(k+1)^{\alpha / 2}
$$

uniformly in $u, v \in(0,1)$ and $k \in \mathbb{N}$.

Proof. Without loss of generality we assume that $0<u \leq v<1$. Fix $\alpha \in(-1 / 2,1 / 2)$ and $u, v \in(0,1)$. Note that 3.1 yields

$$
\frac{\left|\varphi_{k}^{\alpha}(s)\right|}{s} \lesssim(k+1)^{1 / 4}+s^{\alpha-1 / 2}(k+1)^{\alpha / 2}, \quad s \in(0,1), k \in \mathbb{N} .
$$


Hence, applying 3.3$),(3.2)$, and using the fact that the function $s \rightarrow s^{\alpha+1 / 2}$ is $(\alpha+1 / 2)$ Hölder continuous on $(0,1)$, we get that

$$
\begin{aligned}
\left|\varphi_{k}^{\alpha}(u)-\varphi_{k}^{\alpha}(v)\right| & =\left|\int_{u}^{v}\left(-2 \sqrt{k} \varphi_{k-1}^{\alpha+1}(s)+\left(\frac{2 \alpha+1}{2 s}-s\right) \varphi_{k}^{\alpha}(s)\right) d s\right| \\
& \lesssim|u-v|(k+1)^{1 / 4}+(k+1)^{\alpha / 2}\left|\int_{u}^{v} s^{\alpha-1 / 2} d s\right| \\
& \lesssim|u-v|(k+1)^{1 / 4}+(k+1)^{\alpha / 2}|u-v|^{\alpha+1 / 2}
\end{aligned}
$$

uniformly in $u, v \in(0,1)$ and $k \in \mathbb{N}$. This finishes the proof.

Lemma 3.3. For $\alpha \in(-1 / 2,1 / 2)$ the estimate

$$
\left\|u^{-1} R_{r}^{\alpha}(u, \cdot)\right\|_{L^{2}\left(\mathbb{R}_{+}\right)} \lesssim(1-r)^{-3 / 4}+u^{\alpha-1 / 2}(1-r)^{-(\alpha+1) / 2}
$$

holds uniformly in $r \in(1 / 2,1)$ and $u>0$.

Proof. Fix $\alpha \in(-1 / 2,1 / 2)$. Using (3.6) and the estimates (see [8, p. 136])

$$
\begin{array}{ll}
I_{\nu}(s) \lesssim s^{\nu}, & s \in(0,1), \\
I_{\nu}(s) \lesssim s^{-1 / 2} e^{s}, & s \in(1, \infty),
\end{array}
$$

we obtain the pointwise bound (compare [12, (8)])

$$
R_{r}^{\alpha}(u, v) \lesssim \begin{cases}(1-r)^{-\alpha-1}(u v)^{\alpha+1 / 2} \exp \left(-\frac{1+r}{2(1-r)}\left(u^{2}+v^{2}\right)\right) & \text { if } v \leq(1-r) /(2 \sqrt{r} u) \\ (1-r)^{-1 / 2} \exp \left(-\frac{1+r}{2(1-r)}(v-u)^{2}\right) & \text { if } v \geq(1-r) /(2 \sqrt{r} u)\end{cases}
$$

Now we shall prove the claim. The following estimates are uniform in $r \in(1 / 2,1)$ and in the indicated ranges of $u$. Note first that for $u>0$,

$$
\begin{aligned}
\int_{0}^{\frac{1-r}{2 \sqrt{r} u}} u^{-2} R_{r}^{\alpha}(u, v)^{2} d v & \lesssim(1-r)^{-2(\alpha+1)} \int_{0}^{\frac{1-r}{2 \sqrt{r} u}} u^{2 \alpha-1} v^{2 \alpha+1} \exp \left(-\frac{1+r}{1-r} v^{2}\right) d v \\
& \lesssim(1-r)^{-(\alpha+1)} u^{2 \alpha-1} \int_{0}^{\infty} v^{2 \alpha+1} e^{-v^{2}} d v \\
& \lesssim(1-r)^{-(\alpha+1)} u^{2 \alpha-1}
\end{aligned}
$$

Secondly, for $u \leq(1-r) /(4 \sqrt{r} u)$, we have

$$
\begin{aligned}
\int_{\frac{1-r}{2 \sqrt{r} u}}^{\infty} u^{-2} R_{r}^{\alpha}(u, v)^{2} d v & \lesssim(1-r)^{-3} \int_{\frac{1-r}{2 \sqrt{r} u}}^{\infty} v^{2} \exp \left(-\frac{1+r}{1-r}(v-u)^{2}\right) d v \\
& \lesssim(1-r)^{-3} \int_{\frac{1-r}{2 \sqrt{r} u}-u}^{\infty}(v+u)^{2} \exp \left(-\frac{1+r}{1-r} v^{2}\right) d v \\
& \lesssim(1-r)^{-3} \int_{0}^{\infty} v^{2} \exp \left(-\frac{1+r}{1-r} v^{2}\right) d v \\
& \lesssim(1-r)^{-3 / 2}
\end{aligned}
$$


and for $u \geq(1-r) /(4 \sqrt{r} u)$ we obtain

$$
\begin{aligned}
\int_{\frac{1-r}{2 \sqrt{r} u}}^{\infty} u^{-2} R_{r}^{\alpha}(u, v)^{2} d v & \lesssim(1-r)^{-1} \int_{\frac{1-r}{2 \sqrt{r} u}}^{\infty}(1-r)^{-1} \exp \left(-\frac{1+r}{1-r}(v-u)^{2}\right) d v \\
& \lesssim(1-r)^{-2} \int_{-\infty}^{\infty} \exp \left(-\frac{1+r}{1-r} v^{2}\right) d v \\
& \lesssim(1-r)^{-3 / 2} .
\end{aligned}
$$

This finishes the proof of the lemma.

Proof of Proposition 3.1. For $\alpha \in\{-1 / 2\} \cup[1 / 2, \infty)$ the claim follows from 12, Proposition 3.4], hence, from now on, we consider only $\alpha \in(-1 / 2,1 / 2)$. Also, without loss of generality, we assume $u \leq u^{\prime}$.

First, note that using the mean value theorem, Parseval's identity, and 3.5 we obtain

$$
\begin{aligned}
\left\|R_{r}^{\alpha}(u, \cdot)-R_{r}^{\alpha}\left(u^{\prime}, \cdot\right)\right\|_{L^{2}\left(\mathbb{R}_{+}\right)} & \leq\left|u-u^{\prime}\right| \sup _{\xi \geq 1 / 2}\left\|\partial_{u} R_{r}^{\alpha}(\xi, \cdot)\right\|_{L^{2}\left(\mathbb{R}_{+}\right)} \\
& \lesssim\left|u-u^{\prime}\right|\left(\sum_{k=0}^{\infty} 2^{-2 k}(k+1)^{5 / 6}\right)^{1 / 2} \\
& \lesssim\left|u-u^{\prime}\right|
\end{aligned}
$$

uniformly in $r \in(0,1 / 2]$ and $u, u^{\prime} \geq 1 / 2$. On the other hand, applying (3.4) and Lemma 3.2, we receive

$$
\left\|R_{r}^{\alpha}(u, \cdot)-R_{r}^{\alpha}\left(u^{\prime}, \cdot\right)\right\|_{L^{2}\left(\mathbb{R}_{+}\right)} \lesssim \sum_{k=0}^{\infty} 2^{-k}\left|\varphi_{k}^{\alpha}(u)-\varphi_{k}^{\alpha}\left(u^{\prime}\right)\right| \lesssim\left|u-u^{\prime}\right|+\left|u-u^{\prime}\right|^{\alpha+1 / 2}
$$

uniformly in $r \in(0,1 / 2]$ and $u, u^{\prime} \in(0,1)$. Combining the above gives the claim for $r \in(0,1 / 2]$.

Now we assume that $r \in(1 / 2,1)$. Invoking the formula (see [8, p. 110])

$$
\frac{d}{d u} I_{\alpha}(u)=\frac{\alpha}{u} I_{\alpha}(u)+I_{\alpha+1}(u)
$$

we get

$$
\partial_{u} R_{r}^{\alpha}(u, v)=\left(\frac{2 \alpha+1}{2 u}-\frac{1+r}{1-r} u\right) R_{r}^{\alpha}(u, v)+\frac{2 r v}{1-r} R_{r}^{\alpha+1}(u, v) .
$$

Using [13, Lemma 3.2] (originally from [11, pp. 6-7]) we obtain

$$
\left|\frac{2 r v}{1-r} R_{r}^{\alpha+1}(u, v)-\frac{1+r}{1-r} u R_{r}^{\alpha}(u, v)\right| \lesssim \frac{1}{u} R_{r}^{\alpha+1}(u, v)+\left(u+\frac{v-u}{1-r}\right) R_{r}^{\alpha}(u, v)
$$

uniformly in $r \in(1 / 2,1), u, v>0$. Proceeding as in the proof of [12, Proposition 3.4] one can show that

$$
\left\|\frac{1}{u} R_{r}^{\alpha+1}(u, \cdot)+\left(u+\frac{\cdot-u}{1-r}\right) R_{r}^{\alpha}(u, \cdot)\right\|_{L^{2}\left(\mathbb{R}_{+}\right)} \lesssim(1-r)^{-3 / 4}
$$


uniformly in $r \in(1 / 2,1)$ and $u>0$. We leave the details for the interested reader. Thus, we arrived at

$$
\begin{aligned}
\left\|R_{r}^{\alpha}(u, \cdot)-R_{r}^{\alpha}\left(u^{\prime}, \cdot\right)\right\|_{L^{2}\left(\mathbb{R}_{+}\right)} & =\left\|\int_{u}^{u^{\prime}} \partial_{s} R_{r}^{\alpha}(s, \cdot) d s\right\|_{L^{2}\left(\mathbb{R}_{+}\right)} \\
& \lesssim \frac{\left|u-u^{\prime}\right|}{(1-r)^{3 / 4}}+\left\|\int_{u}^{u^{\prime}} \frac{2 \alpha+1}{2 s} R_{r}^{\alpha}(s, \cdot) d s\right\|_{L^{2}\left(\mathbb{R}_{+}\right)}
\end{aligned}
$$

uniformly in $r \in(1 / 2,1)$ and $u, u^{\prime}>0$.

In order to complete the proof it suffices to estimate the remaining component. Using Minkowski's integral inequality and Lemma 3.3 we get

$$
\begin{aligned}
\left\|\int_{u}^{u^{\prime}} \frac{2 \alpha+1}{2 s} R_{r}^{\alpha}(s, \cdot) d s\right\|_{L^{2}\left(\mathbb{R}_{+}\right)} & \lesssim \int_{u}^{u^{\prime}}\left\|s^{-1} R_{r}^{\alpha}(s, \cdot)\right\|_{L^{2}\left(\mathbb{R}_{+}\right)} d s \\
& \lesssim\left|u-u^{\prime}\right|(1-r)^{-3 / 4}+(1-r)^{-(\alpha+1) / 2} \int_{u}^{u^{\prime}} s^{\alpha-1 / 2} d s
\end{aligned}
$$

uniformly in $r \in(1 / 2,1)$ and $u, u^{\prime}>0$. Finally,

$$
\int_{u}^{u^{\prime}} s^{\alpha-1 / 2} d s=\int_{\left(u, u^{\prime}\right) \cap(0,1)} s^{\alpha-1 / 2} d s+\int_{\left(u, u^{\prime}\right) \cap(1, \infty)} s^{\alpha-1 / 2} d s \lesssim\left|u-u^{\prime}\right|^{\alpha+1 / 2}+\left|u-u^{\prime}\right|
$$

uniformly in $r \in(1 / 2,1)$ and $u, u^{\prime}>0$.

Combining the above gives the claim.

Theorem 3.4. For $\alpha \in[-1 / 2, \infty)^{d}$ the inequality

$$
\sum_{n \in \mathbb{N}^{d}} \frac{\left|\left\langle f, \varphi_{n}^{\alpha}\right\rangle\right|}{(|n|+1)^{3 d / 4}} \lesssim\|f\|_{H^{1}\left(\mathbb{R}_{+}^{d}\right)}
$$

holds uniformly in $f \in H^{1}\left(\mathbb{R}_{+}^{d}\right)$. The result is sharp in the sense that for any $\varepsilon>0$ there exists $f \in H^{1}\left(\mathbb{R}_{+}^{d}\right)$ such that

$$
\sum_{n \in \mathbb{N}^{d}} \frac{\left|\left\langle f, \varphi_{n}^{\alpha}\right\rangle\right|}{(|n|+1)^{3 d / 4-\varepsilon}}=\infty
$$

Proof. For the first part of the theorem it suffices to use Proposition 3.1, [12, Lemma 3.1], and Theorem 2.2 ,

In order to prove sharpness, for a given $K \in \mathbb{N}$, we shall construct an appropriate $H^{1}\left(\mathbb{R}_{+}^{d}\right)$-atom a such that

$$
\sum_{n \in \mathbb{N}^{d}}^{\infty} \frac{\left|\left\langle\mathbf{a}, \varphi_{n}^{\alpha}\right\rangle\right|}{(|n|+1)^{3 d / 4-\varepsilon}} \gtrsim K^{\varepsilon}
$$


We begin with the case $d=1$ and $\alpha>-1 / 2$.

First, note that for $\varphi_{k}^{\alpha}$ we have the estimate (compare [10, pp. 435, 453)])

$$
A k^{\alpha / 2} u^{\alpha+1 / 2} \leq \varphi_{k}^{\alpha}(u) \leq B k^{\alpha / 2} u^{\alpha+1 / 2}, \quad 0<u \leq \frac{c}{\sqrt{k}},
$$

where $A, B, c>0$ are constants depending only on $\alpha$.

Fix $\alpha>-1 / 2, \varepsilon>0$, and $K \in \mathbb{N}$. For $\delta \in(0,1 / 2)$ we define

$$
a(u)= \begin{cases}\delta c^{-1}(1-\delta)^{-1} K^{1 / 2} & \text { if } u \in\left(c \delta K^{-1 / 2}, c K^{-1 / 2}\right), \\ -c^{-1} K^{1 / 2} & \text { if } u \in\left(0, c \delta K^{-1 / 2}\right) .\end{cases}
$$

It is easy to check that $a$ is an $H^{1}\left(\mathbb{R}_{+}\right)$-atom. We estimate

$$
\begin{aligned}
\int_{\mathbb{R}_{+}} a(u) \varphi_{k}^{\alpha}(u) d u & \geq \frac{\delta A K^{1 / 2} k^{\alpha / 2}}{c(1-\delta)} \int_{c \delta K^{-1 / 2}}^{c K^{-1 / 2}} u^{\alpha+1 / 2} d u-\frac{K^{1 / 2} B k^{\alpha / 2}}{c} \int_{0}^{c \delta K^{-1 / 2}} u^{\alpha+1 / 2} d u \\
& =\frac{2 k^{\alpha / 2} A \delta}{(2 \alpha+3) c(1-\delta) K^{\alpha / 2+1 / 4}}\left(1-\delta^{\alpha+1 / 2}(\delta+B / A)+\delta^{\alpha+3 / 2} B / A\right) \\
& \gtrsim \frac{k^{\alpha / 2} \delta}{K^{\alpha / 2+1 / 4}(1-\delta)}\left(1-\delta^{\alpha+1 / 2}(1+B / A)\right) .
\end{aligned}
$$

Choosing $\delta$ sufficiently small and independently of $K$ we obtain

$$
\left\langle a, \varphi_{k}^{\alpha}\right\rangle \gtrsim k^{\alpha / 2} K^{-\alpha / 2-1 / 4}
$$

Thus,

$$
\sum_{k=0}^{\infty} \frac{\left|\left\langle a, \varphi_{k}^{\alpha}\right\rangle\right|}{(k+1)^{3 / 4-\varepsilon}} \gtrsim K^{-\alpha / 2-1 / 4} \sum_{k=1}^{K} k^{\alpha / 2+\varepsilon-3 / 4} \gtrsim K^{\varepsilon},
$$

which finishes the proof for $d=1$ and $\alpha>-1 / 2$.

Note that if $\alpha=-1 / 2$, then by (3.3) and (3.1) we have

$$
-\frac{d}{d u} \varphi_{k}^{-1 / 2}(u) \gtrsim k^{3 / 4} u, \quad 0<u \leq \frac{c}{\sqrt{k}} .
$$

Hence, using the mean value theorem we obtain for $k \leq K$,

$$
\begin{aligned}
& \int_{B} a(u) \varphi_{k}^{-1 / 2}(u) d u \\
= & \int_{0}^{c K^{-1 / 2}} a(u)\left(u-\delta c K^{-1 / 2}\right) \frac{d \varphi_{k}^{-1 / 2}}{d u}\left(\xi_{u}\right) d u \\
= & c^{-1} \sqrt{K} \int_{0}^{c K^{-1 / 2}}\left(\delta(1-\delta)^{-1} \mathbb{1}_{B_{1}}(u)+\mathbb{1}_{B_{2}}(u)\right)\left|u-\delta c K^{-1 / 2}\right|\left(-\frac{d \varphi_{k}^{-1 / 2}}{d u}\left(\xi_{u}\right)\right) d u \\
\gtrsim & c^{-1 \sqrt{K}} k^{3 / 4} \delta(1-\delta)^{-1} \int_{0}^{\delta c K^{-1 / 2}}\left(\delta c K^{-1 / 2}-u\right) u d u \\
\simeq & c^{2} K^{-1} k^{3 / 4} \delta^{3}(1-\delta)^{-1} \\
\gtrsim & K^{-1} k^{3 / 4}
\end{aligned}
$$


where $\xi_{u}$ is between $u$ and $\delta c K^{-1 / 2}$.

In the multi-dimensional case we define

$$
\mathbf{a}(x)=\prod_{i=1}^{d} a\left(x_{i}\right), \quad x \in \mathbb{R}_{+}^{d} .
$$

It can be checked that a is an $H^{1}\left(\mathbb{R}_{+}^{d}\right)$-atom and that 3.7 holds. We leave the details for the interested reader.

\section{Generalized Hermite functions}

The generalized Hermite functions of order $\lambda \geq 0$ on $\mathbb{R}$ are defined by the relation

$$
h_{2 k}^{\lambda}(u)=(-1)^{k} 2^{-1 / 2} \varphi_{k}^{\lambda-1 / 2}(|u|), \quad h_{2 k+1}^{\lambda}(u)=(-1)^{k} 2^{-1 / 2} \operatorname{sgn}(u) \varphi_{k}^{\lambda+1 / 2}(|u|), \quad u \in \mathbb{R},
$$

(for $u=0$ we naturally extend the definition of $\varphi_{k}^{\alpha}$ ). In the case $d \geq 1$ we define them as tensor products of the one-dimensional $h_{k}^{\lambda}$. Note that if $\lambda=\mathbf{0}$, then the functions $\left\{h_{n}^{\mathbf{0}}\right\}_{n \in \mathbb{N}^{d}}$ are the classical Hermite functions.

In the following theorem we use two inner products: in $L^{2}\left(\mathbb{R}^{d}\right)$ and in $L^{2}\left(\mathbb{R}_{+}^{d}\right)$ denoted by $\langle\cdot, \cdot\rangle$ and $\langle\cdot, \cdot\rangle_{+}$, respectively.

Theorem 4.1. Let $\lambda \in[0, \infty)^{d}$. The following inequality holds

$$
\sum_{n \in \mathbb{N}^{d}} \frac{\left|\left\langle f, h_{n}^{\lambda}\right\rangle\right|}{(|n|+1)^{3 d / 4}} \lesssim\|f\|_{H^{1}\left(\mathbb{R}^{d}\right)}
$$

uniformly in $f \in H^{1}\left(\mathbb{R}^{d}\right)$. The exponent is sharp, in the sense that for every $\varepsilon>0$ there exists $f \in H^{1}\left(\mathbb{R}^{d}\right)$ such that

$$
\sum_{n \in \mathbb{N}^{d}} \frac{\left|\left\langle f, h_{n}^{\lambda}\right\rangle\right|}{(|n|+1)^{3 d / 4-\varepsilon}}=\infty .
$$

Proof. We shall justify that the claims follow from Theorem 3.4 .

We introduce a function $\mathfrak{m}: \mathbb{N}^{d} \rightarrow\{0,1\}^{d}$ defined by

$$
\mathfrak{m}(n)_{i}=n_{i} \quad(\bmod 2), \quad i=1, \ldots, d
$$

Fix $\lambda \in[0, \infty)^{d}$. For $\eta \in\{0,1\}^{d}$ we shall denote

$$
\lambda_{\eta}=\left(\lambda_{1}-\frac{(-1)^{\eta_{1}}}{2}, \ldots, \lambda_{d}-\frac{(-1)^{\eta_{d}}}{2}\right)
$$

Note that $h_{n}^{\lambda}$ is $\mathfrak{m}(n)$-symmetric. Hence,

$$
\left\langle f, h_{n}^{\lambda}\right\rangle \simeq\left\langle f_{\mathfrak{m}(n)}^{+}, \varphi_{\lfloor n / 2\rfloor}^{\lambda_{\mathfrak{m}(n)}}\right\rangle_{+}, \quad f \in H^{1}\left(\mathbb{R}^{d}\right), n \in \mathbb{N}^{d} .
$$


Thus, we estimate using 2.3

$$
\begin{aligned}
\sum_{n \in \mathbb{N}^{d}} \frac{\left|\left\langle f, h_{n}^{\lambda}\right\rangle\right|}{(|n|+1)^{3 d / 4}} & \simeq \sum_{\eta \in\{0,1\}^{d}} \sum_{\mathfrak{m}(n)=\eta} \frac{\left|\left\langle f_{\eta}^{+}, \varphi_{\lfloor n / 2\rfloor}^{\lambda_{\eta}}\right\rangle_{+}\right|}{(|n|+1)^{3 d / 4}} \leq \sum_{\eta \in\{0,1\}^{d}} \sum_{n \in \mathbb{N}^{d}} \frac{\left|\left\langle f_{\eta}^{+}, \varphi_{n}^{\lambda_{\eta}}\right\rangle_{+}\right|}{(|n|+1)^{3 d / 4}} \\
& \lesssim \sum_{\eta \in\{0,1\}^{d}}\left\|f_{\eta}^{+}\right\|_{H^{1}\left(\mathbb{R}_{+}^{d}\right)} \lesssim\|f\|_{H^{1}\left(\mathbb{R}^{d}\right)} .
\end{aligned}
$$

This finishes the verification of the first claim.

In order to prove the second claim, we fix $\varepsilon>0$. Let $\alpha=\lambda-\mathbf{1} / \mathbf{2}$. Theorem 3.4 yields that there exists $g \in H^{1}\left(\mathbb{R}_{+}^{d}\right)$ such that

$$
\sum_{n \in \mathbb{N}^{d}} \frac{\left|\left\langle g, \varphi_{n}^{\alpha}\right\rangle_{+}\right|}{(|n|+1)^{3 d / 4-\varepsilon}}=\infty
$$

We extend $g$ to an $\mathbf{0}$-symmetric function $f$. We emphasise that $f \in H^{1}\left(\mathbb{R}^{d}\right)$. Hence,

$$
\sum_{n \in \mathbb{N}^{d}} \frac{\left|\left\langle f, h_{n}^{\lambda}\right\rangle\right|}{(|n|+1)^{3 d / 4-\varepsilon}} \geq \sum_{\mathfrak{m}(n)=\mathbf{0}} \frac{\left|\left\langle f, h_{n}^{\lambda}\right\rangle\right|}{(|n|+1)^{3 d / 4-\varepsilon}} \simeq \sum_{n \in \mathbb{N}^{d}} \frac{\left|\left\langle g, \varphi_{n}^{\lambda-\mathbf{1} / \mathbf{2}}\right\rangle_{+}\right|}{\left(2^{d}|n|+1\right)^{3 d / 4-\varepsilon}}=\infty .
$$

This finishes the proof of the theorem.

Theorem 4.1 holds for the classical Hermite functions (that is for $\lambda=\mathbf{0}$ ), and hence the admissible exponent obtained in 9,15 is sharp.

In the previous articles (see 12,13$]$ ) we proved the $L^{1}$-analogues of Hardy's type inequalities. Therefore we present a corresponding result for the generalized Hermite functions below. It can be proved basing on [12, Theorem 5.1] and using similar arguments as in the proof of Theorem 4.1 .

Theorem 4.2. Let $\varepsilon>0$ and $\lambda \in[0, \infty)^{d}$. Then

$$
\sum_{n \in \mathbb{N}^{d}} \frac{\left|\left\langle f, h_{n}^{\lambda}\right\rangle\right|}{(|n|+1)^{3 d / 4+\varepsilon}} \lesssim\|f\|_{L^{1}\left(\mathbb{R}^{d}\right)}
$$

uniformly in $f \in L^{1}\left(\mathbb{R}^{d}\right)$. The result is sharp in the sense that there is $f \in L^{1}\left(\mathbb{R}^{d}\right)$ such that

$$
\sum_{n \in \mathbb{N}^{d}} \frac{\left|\left\langle f, h_{n}^{\lambda}\right\rangle\right|}{(|n|+1)^{3 d / 4}}=\infty
$$

\section{Acknowledgments}

The paper is a part of author's doctoral thesis written under the supervision of Professor Krzysztof Stempak. The author would like to express his gratitude to Professor Stempak for his remarks. This research was supported by the National Science Centre of Poland, NCN grant no. 2018/29/N/ST1/02424. 


\section{References}

[1] R. Askey and S. Wainger, Mean convergence of expansions in Laguerre and Hermite series, Amer. J. Math. 87 (1965), 695-708.

[2] R. Balasubramanian and R. Radha, Hardy-type inequalities for Hermite expansions, JIPAM. J. Inequal. Pure Appl. Math. 6 (2005), no. 1, Article 12, 4 pp.

[3] R. R. Coifman and G. Weiss, Extensions of Hardy spaces and their use in analysis, Bull. Amer. Math. Soc. 83 (1977), no. 4, 569-645.

[4] G. H. Hardy and J. E. Littlewood, Some new properties of fourier constants, Math. Ann. 97 (1927), no. 1, 159-209.

[5] Y. Kanjin, Hardy's inequalities for Hermite and Laguerre expansions, Bull. London Math. Soc. 29 (1997), no. 3, 331-337.

[6] _ Hardy's inequalities for Hermite and Laguerre expansions revisited, J. Math. Soc. Japan 63 (2011), no. 3, 753-767.

[7] Y. Kanjin and K. Sato, Hardy's inequality for Jacobi expansions, Math. Inequal. Appl. 7 (2004), no. 4, 551-555.

[8] N. N. Lebedev, Special Functions and Their Applications, Dover, New York, 1972.

[9] Z. Li, Y. Yu and Y. Shi, The Hardy inequality for Hermite expansions, J. Fourier Anal. Appl. 21 (2015), no. 2, 267-280.

[10] B. Muckenhoupt, Mean convergence of Hermite and Laguerre series II, Trans. Amer. Math. Soc. 147 (1970), 433-460.

[11] I. Nåsell, Rational bounds for ratios of modified Bessel functions, SIAM J. Math. Anal. 9 (1978), no. 1, 1-11.

[12] P. Plewa, Hardy's inequality for Laguerre expansions of Hermite type, Accepted in J. Fourier Anal. Appl. (2018), 19 pp.

[13] _ Sharp Hardy's type inequality for Laguerre expansions, preprint (2018), arXiv:1810.08138.

[14] R. Radha, Hardy-type inequalities, Taiwanese J. Math. 4 (2000), no. 3, 447-456.

[15] R. Radha and S. Thangavelu, Hardy's inequalities for Hermite and Laguerre expansions, Proc. Amer. Math. Soc. 132 (2004), no. 12, 3525-3536. 
[16] M. Satake, Hardy's inequalities for Laguerre expansions, J. Math. Soc. Japan 52 (2000), no. 1, 17-24.

[17] E. M. Stein, Harmonic Analysis: Real-variable Methods, Orthogonality, and Oscillatory Integrals, Princeton Mathematical Series 43, Monographs in Harmonic Analysis III, Princeton University Press, Princeton, NJ, 1993.

[18] K. Stempak, Heat-diffusion and Poisson integrals for Laguerre expansions, Tohoku Math. J. (2) 46 (1994), no. 1, 83-104.

[19] G. Szegö, Orthogonal polynomials, Fourth edition, American Mathematical Society, Colloquium Publication XXIII, American Mathematical Society, Providence, R.I., 1975 .

Paweł Plewa

Faculty of Pure and Applied Mathematics, Wrocław University of Science and Technology, Wyb. Wyspiańskiego 27, 50-370 Wrocław, Poland

E-mail address: pawel.plewa@pwr.edu.pl 